\section{Mapeamento de áreas de risco à saúde pública por meio de métodos geoestatísticos}

\author{
Public health risk maps using \\ geostatistical methods
}

Roberto Wagner Lourenço 1,2 Paulo Milton Barbosa Landim 2

\footnotetext{
1 Unidade Diferenciada de Sorocaba/Iperó, Universidade Estadual Paulista Júlio de Mesquita Filho, Sorocaba, Brasil. 2 Departamento de Geologia Aplicada, Universidade Estadual Paulista Júlio de Mesquita Filho, Rio Claro, Brasil.

Correspondência R. W. Lourenço Unidade Diferenciada de Sorocaba/Iperó, Universidade Estadual Paulista Júlio de Mesquita Filho. Av. 3 de março 511 Sorocaba, SP 13506-900, Brasil. robertow@sorocaba.unesp.br
}

\begin{abstract}
The purpose of this paper was to demonstrate an application of geostatistical methods to public health risk maps through the identification of areas with elevated concentrations of heavy metals. The study focused on the element lead $\mathrm{Pb}$ ) from aerial transportation or loading of particles due to soil leaching in an area with major urban and industrial concentration in the Baixada Santista on the coastland of São Paulo State, Brazil. Maps with the spatial distribution of lead were produced using ordinary kriging; subsequently indicative kriging was performed to identify soil sites with contamination levels higher than the maximum acceptable level defined by the São Paulo State Environmental Control Agency. The resulting maps showed areas with increased probability of public health risk. The methodology proved to be a promising approach for decision-making related to health public policies and environmental planning.
\end{abstract}

Heavy Metals; Risk Map; Soil Pollutants

\section{Introdução}

O solo atua como um filtro, tendo a capacidade de depurar grande parte das impurezas nele depositadas. No entanto, essa capacidade é limitada, podendo ocorrer alteração da qualidade do solo em virtude do efeito cumulativo da deposição de poluentes atmosféricos, da aplicação de defensivos agrícolas e fertilizantes e da disposição de resíduos sólidos industriais, urbanos, materiais tóxicos e radioativos 1 .

A Companhia de Tecnologia e Saneamento Básico do Estado de São Paulo (CETESB) 2, por meio de testes laboratoriais e pesquisas de campo, elaborou uma lista de valores orientadores para concentração de determinados metais pesados no solo. Essa lista é utilizada como referência para identificação de áreas consideradas já contaminadas ou suspeitas de contaminação, as quais venham a oferecer risco à saúde pública. Resumidamente, os valores são divididos em teores de referência, valores de alerta e valores de intervenção.

Teores de referência indicam o limite de qualidade para um solo considerado próprio e devem ser utilizados em ações de prevenção da poluição no controle de áreas contaminadas. Foram estabelecidos com base em análises químicas dos diversos tipos de solos do Estado de São Paulo e, por exemplo, para o chumbo $(\mathrm{Pb})$, o valor de referência não pode ultrapassar $17 \mathrm{mg} . \mathrm{kg}^{-1}$. 
Valor de alerta indica uma possível alteração da qualidade natural dos solos e é utilizado em caráter preventivo quando excedido. Devese, também, exigir o monitoramento das águas subterrâneas, identificando-se e controlandose as fontes de poluição. Foi derivado para metais, com base em revisão bibliográfica sobre fitotoxicidade. Para o metal pesado $\mathrm{Pb}$, esse valor se encontra acima de $17 \mathrm{mg} . \mathrm{kg}^{-1}$ e inferior a 100mg.kg-1.

Valor de intervenção indica o limite de contaminação do solo, acima do qual existe risco potencial à saúde humana, e é utilizado em caráter corretivo no gerenciamento de áreas contaminadas. Quando excedido, requer alguma forma de intervenção na área avaliada, de forma a interceptar as vias de exposição, devendo ser efetuada uma avaliação de risco caso a caso. Para o Pb este valor é superior a $100 \mathrm{mg} . \mathrm{kg}^{-1}$.

$\mathrm{O} \mathrm{Pb}$ é um dos metais pesados de utilização industrial mais variada, presente, sobretudo em baterias. Como todo metal pesado, degrada-se muito lentamente no meio ambiente, persistindo durante décadas no solo e no fundo de rios, lagoas e represas. É comprovadamente carcinogênico, teratogênico e tóxico para o sistema reprodutivo. A presença de quantidades elevadas de $\mathrm{Pb}$ no sangue ocasiona problemas neurológicos, como falta de concentração e dificuldades na fala. Não é metabolizado pelos animais e sofre o processo de bioacumulação, afetando mais os animais do topo da cadeia alimentar, entre os quais está o homem.

Têm-se realizado, por meio de métodos geoestatísticos, diversos estudos relacionados à produção de mapas, que visam à identificação de áreas de risco à saúde pública, considerando a poluição do solo.

Atteia et al. 3 analisaram a distribuição espacial dos metais pesados cádmio (Cd), cobalto $(\mathrm{Co})$, cobre $(\mathrm{Cu})$, níquel $(\mathrm{Ni})$ e $\mathrm{Pb}$ na área do Jura, na Suíça, por meio de técnicas geoestatísticas. Os mapas de Co e Ni obtidos apresentaram um padrão de distribuição indicativo de material resultante de ações geológicas, sugerindo que ambos os metais seriam derivados de rochas matrizes. $\mathrm{O} \mathrm{Cu}$ e $\mathrm{Pb}$ teriam padrões de distribuição que provavelmente estariam associados ao uso de fertilizantes ou adubos utilizados por atividades agrícolas. O Cd poderia estar associado tanto a atividades humanas, tal como fundição, ou aplicação de fertilizantes, quanto a depósitos geológicos específicos, como morainas, formadas pela ação de transporte glacial.
Franssen et al. 4 analisaram a variação de metais pesados no solo em três diferentes profundidades em um espaço tridimensional. Para analisar a variabilidade espacial, foi utilizada a classificação da lógica fuzzy por meio de média k. As concentrações de metais pesados mostraram mudanças abruptas nas diferentes profundidades, com altos coeficientes de variação. Para evitar uma fragmentação no processo de classificação da lógica fuzzy, a variabilidade espacial dos valores de $\mathrm{Cu}, \mathrm{Pb}$ e Zinco (Zn), que apresentaram correlação nos planos horizontal e vertical, foram interpolados por meio da $\mathrm{kri}$ gagem ordinária. Os mapas dos diferentes metais foram integrados em um mapa de classificação final de áreas contaminadas. O efeito da densidade das observações, medido pela precisão de uma classe pertencer à outra, foi explicado pela função de distribuição cumulativa condicional (fdcc) expressa no mapa de incerteza.

Carlon et al. 5 realizaram um trabalho de mapeamento próximo a áreas industriais localizadas ao norte de Fidenza, Parma, na Itália, onde foram detectadas altas concentrações de hidrocarbonetos. Para tanto, empregaram métodos de krigagem para interpolação e análise das componentes principais, a fim de identificar os principais agentes de contaminação do solo. Os resultados mostraram que a geoestatística e a estatística multivariada podem ser muito úteis na determinação de áreas de risco à saúde humana.

Van Meirvenne \& Goovaerts 6 aplicaram um método estatístico não paramétrico para avaliar a probabilidade de ocorrência da concentração de metal pesado no solo, tendo como limite de corte valores específicos em uma região localizada no Nordeste da Bélgica. A metodologia foi aplicada em uma área contaminada por Cd por emissão aérea, e, por intermédio da krigagem indicativa, foi construída uma função de distribuição cumulativa (fdc). O limite de corte, utilizado como parâmetro para os valores de $\mathrm{Cd}$, considerou a concentração de matéria orgânica e argila comparada com a de outros locais para o mesmo tipo de solo. Os mapas gerados mostraram que, em um nível de probabilidade de $80,0 \%$, foram encontrados $27,3 \%$ de área contaminada com os valores acima do limite de corte, ilustrando a extensão da poluição. Adicionalmente, avaliaram-se os locais onde a incerteza sobre a probabilidade mapeada excedeu o limite de corte, a fim de identificar as regiões nas quais deveriam ser coletadas novas amostras de solo para melhorar a qualidade do mapeamento. 
Lin et al. 7 caracterizaram os padrões de distribuição espacial de chumbo em solos, por meio de simulação gaussiana seqüencial e krigagem ordinária, em áreas cultivadas com arroz no norte de Changhua, em Taiwan. O trabalho demonstrou que as técnicas de simulação gaussiana seqüencial apresentaram melhores resultados quando comparadas com a krigagem ordinária.

Lin et al. 8 usaram os métodos krigagem fatorial e krigagem indicativa para analisar a variação espacial de metais pesados em solos agrícolas ao norte de Changhua, em Taiwan. Com o objetivo de auxiliar no monitorando ambiental, foram analisados os padrões de probabilidade de poluição por $\mathrm{Cd}$, cromo $(\mathrm{Cr})$ e $\mathrm{Cu}$, tendo como base propostas de remediação e planejamento. $\mathrm{O}$ estudo mostrou que as variações de $\mathrm{Cd}, \mathrm{Cr}$ e $\mathrm{Cu}$ no solo tiveram forte correlação com os locais de maior proximidade de áreas industriais.

Critto et al. ${ }^{9}$ utilizaram ferramentas de estatísticas multivariadas, como análise das componentes principais e técnicas de geoestatística, para obter a distribuição espacial de contaminantes químicos no solo, nas imediações de uma lagoa próxima de Veneza, na Itália. Os autores avaliaram a migração dos contaminantes no solo nas áreas subjacentes em direção às águas superficiais e do lençol freático. Nesse trabalho, os autores concluíram que muitos dos contaminantes medidos nas áreas mais próximas da lagoa estavam influenciando diretamente a qualidade das águas superficiais e subterrâneas do local.

Juang et al. 10 utilizaram krigagem indicativa e simulação indicativa seqüencial para gerar mapas de poluição levando-se em conta a concentração do Cu em uma área pertencente à cidade de Hsingchu, em Taiwan. Nesse trabalho, os autores procuraram localizar as áreas de maior probabilidade de contaminação por meio da krigagem indicativa, medindo, por meio da simulação, a incerteza do mapeamento.

McGrath et al. ${ }^{11}$ produziram mapas de poluição com base na distribuição espacial do $\mathrm{Pb}$ em Silvermines, República da Irlanda. Os mapas gerados serviram como valiosa informação sobre as áreas de risco à saúde pública e como suporte a decisões e planejamentos.

\section{Material e métodos}

Os estudos relacionados com o comportamento dos metais pesados em solo podem ser associados ao termo risk assessment $12,13,14$, considerando-se que a contaminação do solo, bem como das águas, deve ser definida de acordo com o potencial de risco aos seres humanos e ao meio ambiente.

Assim, como parte de um programa que requeira uma seqüência lógica de atividades e decisões a serem tomadas desde a suspeita da contaminação até o alcance das metas de remediação, faz-se necessária a identificação das áreas que possam apresentar maior probabilidade de risco à saúde pública. Para tal identificação, podem ser utilizadas duas técnicas geoestatísticas: a krigagem ordinária (KO) e a krigagem indicativa (KI), que fornecerão mapas mostrando a distribuição espacial do elemento que se deseja estudar. No presente trabalho foi considerado o teor do metal pesado $\mathrm{Pb}$ no solo.

Para o desenvolvimento desta pesquisa, foi utilizado o programa Surfer 8.0, para o mapeamento da KO, e o Sistema de Informações Geográficas (SIG) Idrisi 32 (http://www.clarklabs. org), para o mapeamento da KI.

\section{Análise geoquímica}

O procedimento analítico executado para obter o metal pesado foi realizado a partir da coleta de solo em 123 pontos de amostragem, localizados nas áreas urbanas e periféricas dos centros administrativos dos municípios de Santos, São Vicente, Cubatão, Praia Grande, Vicente de Carvalho e Guarujá, litoral paulista, em áreas com ou sem influência antropogênica (Figura 1). Após a secagem, o solo foi desagregado e peneirado a $80 \#$, homogeneizado e quarteado. Foi utilizado o método de abertura multiácido $\left(\mathrm{HCl}, \mathrm{HNO}_{3}\right.$, $\mathrm{HF}$ e $\left.\mathrm{HClO}_{4}\right)$, com leitura por Espectometria de Indução em Plasma (ICP Inductively Coupled Plasma) para detecção dos valores de $\mathrm{Pb}$ no solo 15.

\section{Metodologia geoestatística}

A geoestatística calcula estimativas dentro de um contexto regido por um fenômeno natural com distribuição no espaço e, desse modo, supõe que os valores das variáveis, consideradas como regionalizadas, sejam espacialmente correlacionados. Por isso, essa metodologia tem tido grande aplicação, principalmente para efetuar estimativas e simulações de variáveis em locais não amostrados.

De uma forma geral, a metodologia geoestatística procura extrair, de uma aparente aleatoriedade dos dados coletados, as características estruturais probabilísticas do fenômeno regionalizado, ou seja, uma função de correlação entre os valores situados numa determinada vizinhança e direção no espaço amostrado. O método de estimativa básico utilizado é o da 


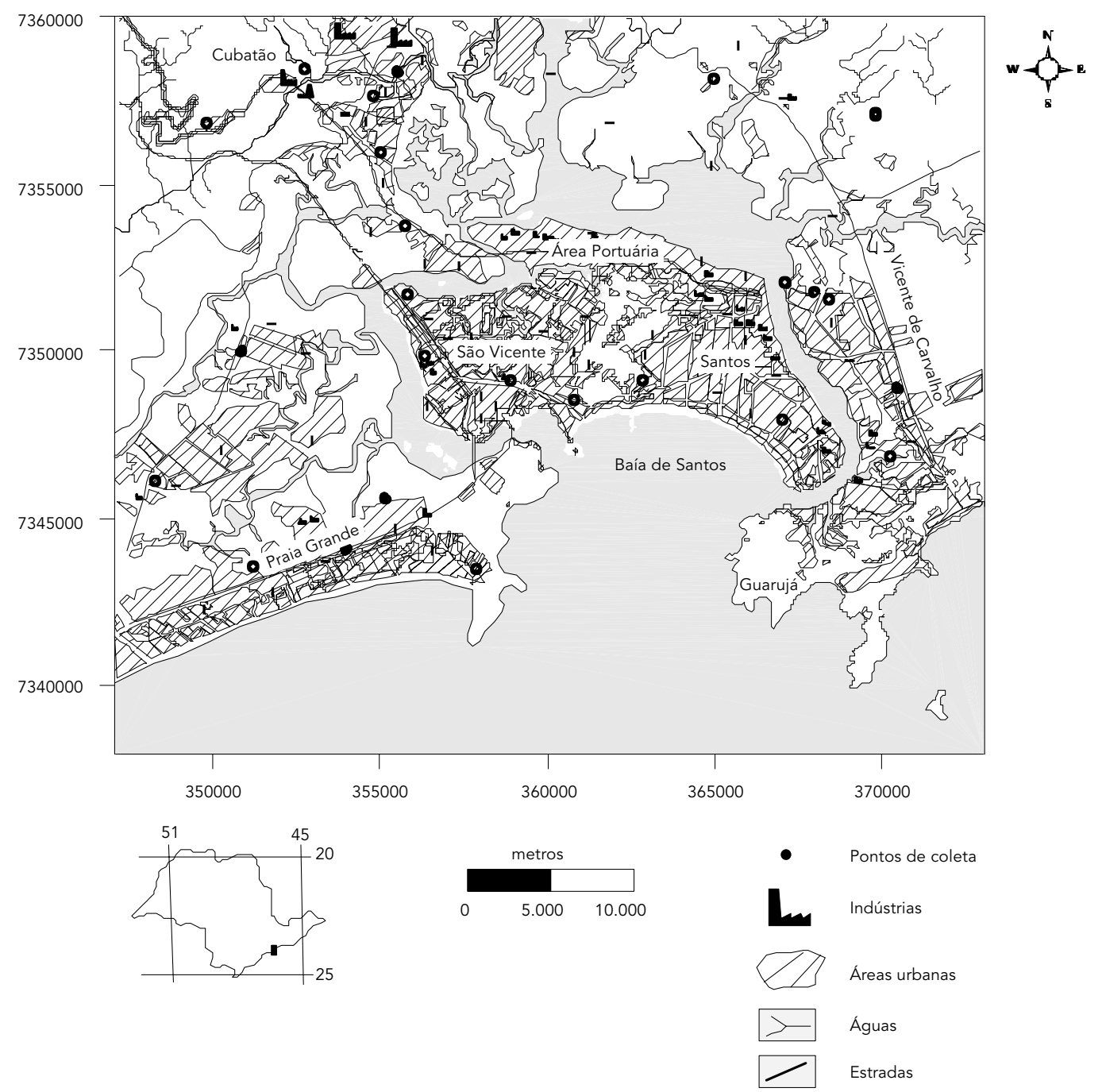

krigagem. Trata-se de um processo de estimativa por médias móveis, de valores de variáveis, distribuídas no espaço a partir de valores adjacentes, considerados como interdependentes por uma função denominada variograma. Como no cálculo dessa função a somatória de diferenças ao quadrado é dividida por 2, a denominação correta seria semivariograma, porém o emprego do termo variograma está consagrado.

Se uma variável regionalizada $v(i)$ for coletada em diversos pontos $i$, o valor de cada ponto estará relacionado com valores obtidos a partir de pontos situados a uma certa distância $h$, e a influência será tanto maior quanto menor for a distância entre os pontos. O grau de relação entre pontos numa certa direção pode ser expresso pela co-variância, sendo os pontos regularmente espaçados por múltiplos inteiros de $h$. O vetor $h$, apresentando-se infinitamente pequeno, faz com que a variância seja mínima e a co-variância máxima. Haverá um valor $h$ para o qual ambas podem apresentar valores aproximadamente iguais, porém, à medida que $h$ aumenta, a co-variância diminui, enquanto a variância aumenta, porque ocorre progressivamente maior independência entre os pontos a distâncias cada vez maiores. A semivariância distribui-se, assim, de 0 , quando $h=0$, até um 
valor maior aproximadamente igual à variância das observações, se os dados forem estacionários, isto é, se não ocorrer a presença de deriva.

Sendo $v(1), v(2), \ldots . v(i), \ldots v(n)$ realizações de uma variável regionalizada, a estimativa não tendenciosa da semivariância é dada por:

$$
\gamma(\mathrm{h})=\frac{1}{2 \mathrm{n}} \Sigma\left(\mathrm{v}_{\mathrm{i}+\mathrm{h}}-\mathrm{v}_{\mathrm{i}}\right)^{2}
$$

Tais relações são mostradas quando a função $\gamma(h)$ é colocada em gráfico contra $\Delta h$ para originar o variograma. Este expressa o comportamento espacial da variável regionalizada e mostra, segundo a Figura 2:

- o alcance (a), que indica a distância a partir da qual as amostras passam a não possuir correlação espacial e a relação entre elas torna-se aleatória; toda amostra cuja distância ao ponto a ser estimada for menor ou igual à amplitude fornece informações sobre o ponto;

- o patamar $(C+C o)$, que indica o valor segundo o qual a função estabiliza-se no campo aleatório, correspondente à distância “a”; mostra a variabilidade máxima entre pares de valores, isto é, a variância dos dados e, conseqüentemente, co-variância nula;

- a continuidade, pela forma do variograma, em que para $\mathrm{h} \cong 0, \gamma(h)$ já apresenta algum valor; essa situação, representada por $C o$, é conhecida como efeito pepita, o qual pode ser atribuído a erros de medição ou ao fato de que os dados não foram coletados a intervalos suficientemente pequenos para mostrar o comportamento espacial subjacente do fenômeno em estudo, isto é, não é capturado um fenômeno numa escala maior;

- a anisotropia, quando os semivariogramas mostram parâmetros diferentes para diferentes direções de amostragem.

A krigagem é entendida como uma série de técnicas de análise de regressão que procura minimizar a variância estimada valendo-se de um modelo prévio, levando em conta a dependência estocástica entre os dados distribuídos no espaço. A forma mais usual é a KO, e é considerado o melhor estimador linear não enviesado (best linear unbiased estimator - BLUE), em que a variância da krigagem é utilizada para definir intervalos de confiança do tipo gaussiano.

A diferença fundamental entre a krigagem e outros métodos de interpolação é a maneira como os pesos são atribuídos às diferentes amostras. Na krigagem, os pesos são determinados a partir de uma análise espacial, com base no semivariograma experimental. Além disso, a krigagem fornece, em média, estimativas não tendenciosas e com variância mínima 16.
Obtido o variograma experimental, torna-se necessário ajustá-lo a uma função que represente a tendência de $\hat{\gamma}(\mathrm{h})$ em relação a h. Isso é feito a fim de modelar fenômenos que possuem capacidade infinita de dispersão. Desse modo, as estimativas, obtidas valendo-se da krigagem, serão mais exatas e, portanto, mais confiáveis.

O procedimento de ajuste não é direto e automático, como no caso de uma análise de regressão linear, por exemplo, mas, sim, interativo, até ser obtido o modelo de variograma julgado satisfatório. Os modelos básicos estão divididos em dois tipos: modelos com patamar e modelos sem patamar. Os modelos com patamar mais utilizados são: modelo esférico (Sph), modelo exponencial (Exp) e modelo gaussiano (Gau), como apresentado na Figura 3, com o mesmo alcance (a).

As variâncias de krigagem, sendo condicionadas apenas pelo arranjo geométrico dos pontos e, portanto, independentes dos valores das amostras, não são normalmente medidas de acurácia da estimativa local. Para satisfazer a essa necessidade, uma das soluções apontadas é a KI. O enfoque passa a ser, neste caso, não estimar um determinado valor, como na KO, mas, sim, definir áreas com maior ou menor probabilidade de que um determinado evento ocorra.

A KI consiste basicamente na aplicação da KO para a variável transformada, ou seja, a variável resultante da aplicação da função não linear $f(z)=0$ ou 1 . O conceito inicial foi apresentado por Journel 17 como uma proposta para construir uma função de distribuição de probabilidades acumuladas (cumulative distribution function) para a estimativa de distribuições espaciais. O conceito da transformação indicativa é dos mais simples e amigáveis, visto que os variogramas indicativos são os mais fáceis de modelar.

No processo básico da krigagem, a estimativa é feita para determinar um valor médio em um local não amostrado. Entretanto, podemse, também, fazer estimativas baseadas em valores que se situam abaixo ou acima de um determinado nível de corte (cutoff). Esse procedimento, estabelecido para diversos níveis de corte (percentis, decis e/ou quartis, por exemplo) de uma distribuição acumulada, conduzirá a uma estimativa de vários valores dessa distribuição em um determinado local, cuja função pode ser ajustada.

Para que esses objetivos sejam atingidos, o primeiro passo, na krigagem indicativa, é transformar os dados originais em indicadores, isto é, transformar os valores que estão acima de um determinado nível de corte em zero (0) e os que estão abaixo, em um (1): 


$$
i_{j}\left(v_{c}\right)=\left\{\begin{array}{l}
1 \text { se } v_{j} \leq v_{c} \\
0 \text { se } v_{j}>v_{c}
\end{array},\right.
$$

$v_{c}=$ nível de corte; $v_{j}=$ valor observado

A freqüência acumulada de valores observados, por exemplo, abaixo do nível de corte pode ser expressa por:

$$
\mathrm{F}\left(\mathrm{v}_{\mathrm{c}}\right)=\frac{1}{\mathrm{n}} \sum_{\mathrm{i}=1}^{\mathrm{n}} \mathrm{i}_{\mathrm{j}}\left(\mathrm{v}_{\mathrm{c}}\right)
$$

De modo idêntico, a proporção de valores abaixo do nível de corte pode ser considerada como a média ponderada dos indicadores, no caso 1 , situados na vizinhança do local avaliado, segundo:

$$
\hat{\mathrm{F}}\left(\mathrm{v}_{\mathrm{c}}\right)=\sum_{\mathrm{j}=1}^{\mathrm{n}} \mathrm{w}_{\mathrm{j}} \cdot \mathrm{i}_{\mathrm{j}}\left(\mathrm{v}_{\mathrm{c}}\right),
$$

em que $w_{j}$ são os pesos, cuja soma deve ser 1 pela condição de não viés; $i_{j}$ são os indicadores e $v_{c}$, o nível de corte. Dessa forma, são calculados os variogramas experimentais indicativos para determinados níveis de corte e estabelecidos os modelos variográficos para os mesmos.

Os variogramas indicativos podem ser estimados pela função:

$$
\gamma_{i}\left(h, v_{c}\right)=\frac{1}{2 N_{h}} \sum_{i=1}^{N h}\left[i\left(x+h, v_{c}\right)-i\left(x, v_{c}\right)\right]^{2}
$$

em que $h=$ passo (lag) básico; $v_{c}=$ nível de corte (cutoff); $N=$ número de pares.

Efetuando-se a KO pontual nos valores transformados, obtém-se a probabilidade de $v_{i}<v_{c}$. $\mathrm{A}$ medida que se incrementar $v_{c}$, obter-se-ão valores estimados da função de distribuição de probabilidades acumuladas, assim expressos:

$$
\mathrm{F}\left(\mathrm{v} ; \frac{\mathrm{v}_{\mathrm{c}}}{\mathrm{n}}\right)=\mathrm{E}\left[\frac{\mathrm{i}\left(\mathrm{v} ; \mathrm{v}_{\mathrm{c}}\right)}{\mathrm{n}}\right]
$$

$\operatorname{com} i\left(v ; v_{c}\right)=1$, se $v_{i}<v_{c .}$.

Definidas as funções da distribuição acumulada, pode-se obter qualquer intervalo probabilístico da variável, ou seja:

$$
F\left(v_{j}\right)-F\left(v_{i}\right)
$$

em que $v_{j}>v_{c}$.

Por fim, de posse dessas proporções para os vários níveis, estabelece-se a função de distribuição acumulada condicionada para os diversos locais de ocorrência da variável sob análise.

Antes de efetuar a KI, é necessário que, para cada nível de corte, seja encontrado um va-
Figura 2

Exemplo de semivariograma e suas propriedades.

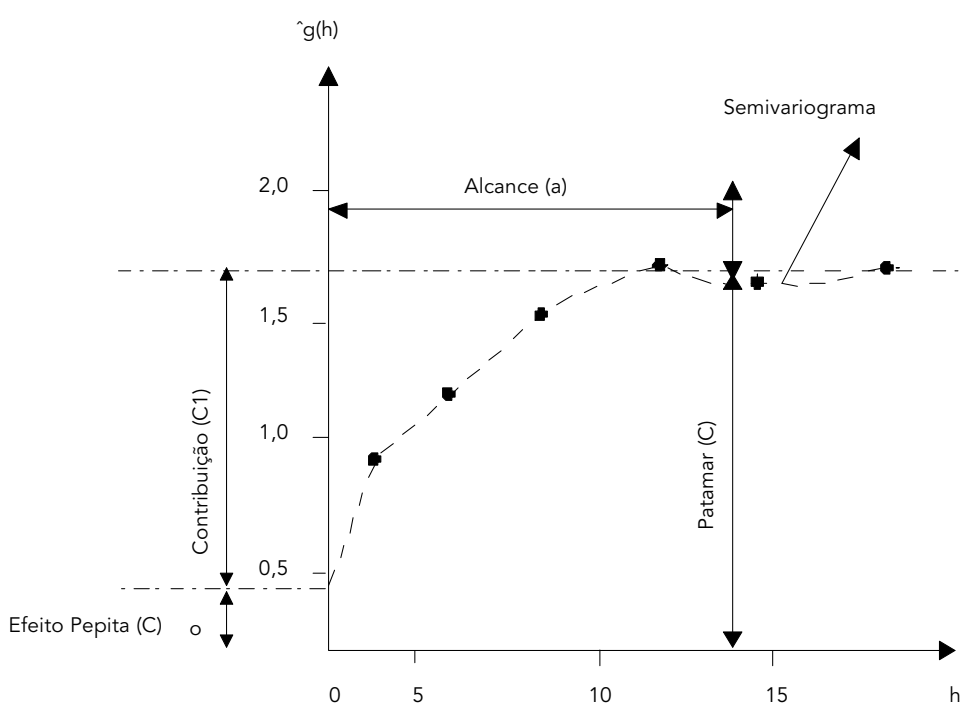

Figura 3

Representação gráfica de modelos com patamar.

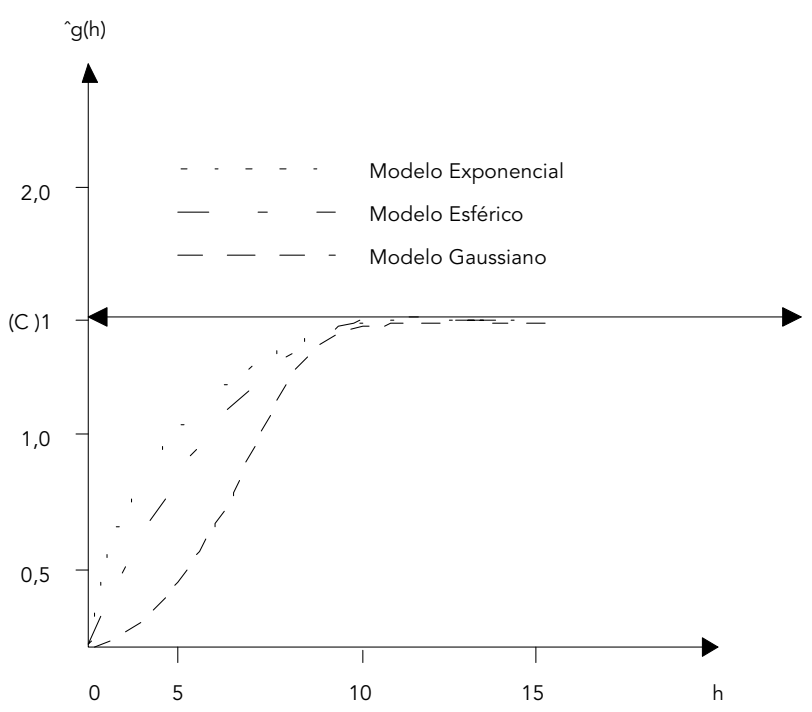


riograma e uma boa aproximação, procurando encontrar, se possível, o mesmo modelo para todos eles, principalmente aquele correspondente à mediana 18.

Exemplos podem ser encontrados em Isaaks \& Srivastava 18; Sturaro \& Landim 19 e Sturaro et al. 20 .

\section{Resultados e discussão}

Em apenas quatro pontos de coleta, foram detectados valores de teores de $\mathrm{Pb}$ inferiores a 17mg.kg-1, ou seja, valores considerados máximos para solos tidos como referência 2 . Em outros 92 pontos de coleta, os valores se mostraram acima dos de referência e inferiores aos de intervenção, ou seja, 100mg.kg-1, indicando um estado de alerta para esses locais. As situa- ções mais graves de contaminação do solo foram encontradas em 27 pontos de coleta, nos quais os valores de concentração do metal pesado $\mathrm{Pb}$, com valores acima de $100 \mathrm{mg} \cdot \mathrm{kg}^{-1}$, indicaram programas de intervenção como forma de minimizar o problema.

\section{Elaboração dos mapas de risco à saúde pública}

Para a elaboração do mapa da distribuição espacial do $\mathrm{Pb}$ e, conseqüentemente, das áreas com maior probabilidade de risco à saúde pública, foram utilizadas as técnicas da $\mathrm{KO}$ e da KI respectivamente.

O procedimento inicial foi a construção de uma superfície variográfica dos valores de $\mathrm{Pb}$ espacialmente distribuídos para detectar as direções preferenciais de variabilidade (Figura 4).

Figura 4

Superfície variográfica dos valores de $\mathrm{Pb}$.

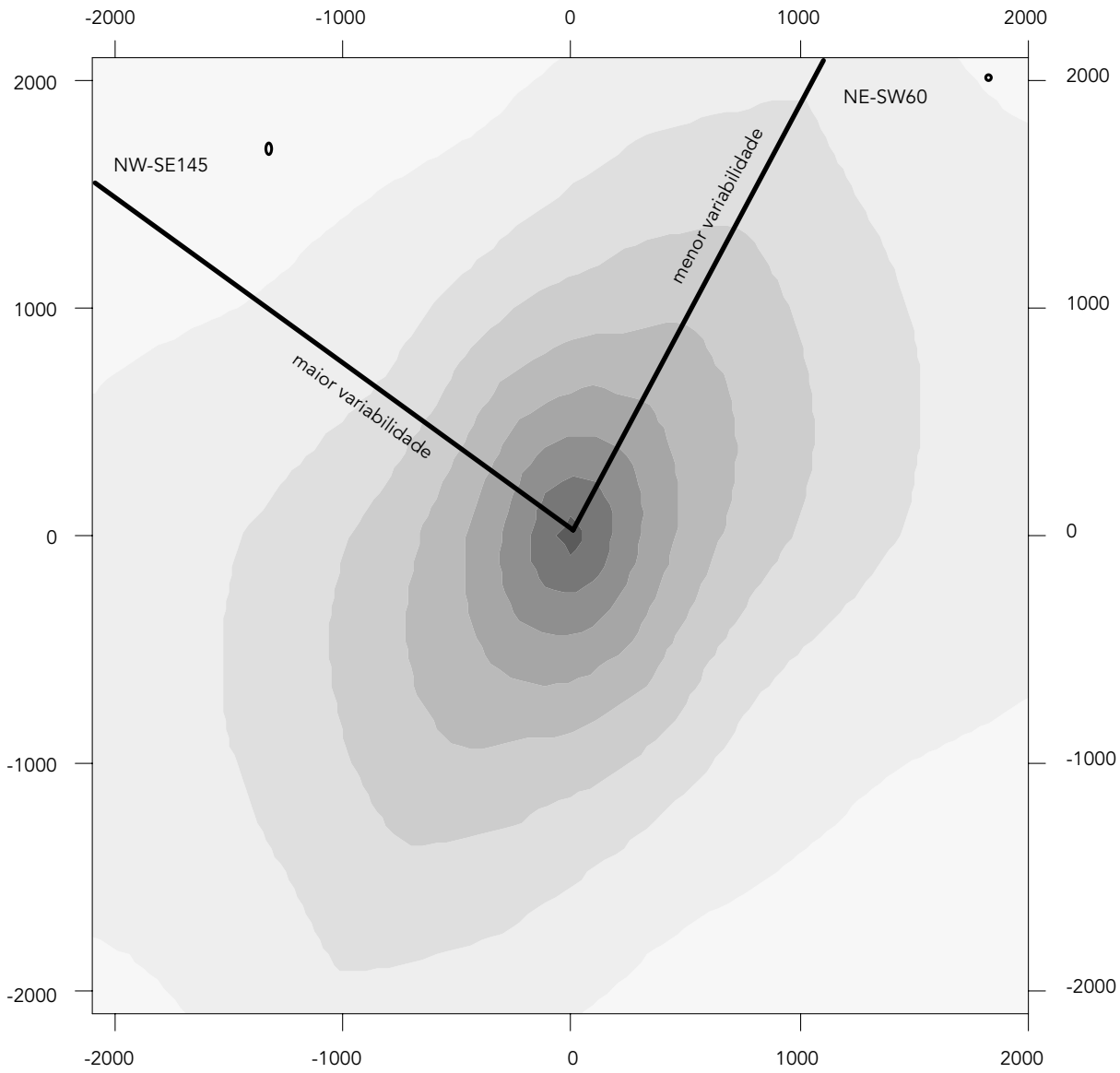


Com base na superfície variográfica, foi calculado o variograma experimental em duas direções distintas. Na direção NE-SW $60^{\circ}$, entendida como a de menor variabilidae, a abertura de tolerância angular foi de 22,5 $5^{\circ}$, com 10 lags e distâncias de $500 \mathrm{~m}$. A este variograma experimental foi ajustado um modelo variográfico teórico exponencial, que é um tipo de curva que atinge o patamar $(C)$ assintoticamente, característico de modelos sem tendência. O método de ajuste utilizado foi o dos mínimos quadrados, e a este gráfico foi ajustado um modelo de curva com efeito pepita igual a 80 , patamar de 475 e alcance de $2.550 \mathrm{~m}$, representado por losangos pretos e linha traço-ponto preta. $\mathrm{Na}$ direção NW-SE $145^{\circ}$, interpretada como a de maior variabilidade, foi igualmente calculado um variograma experimental com abertura de tolerância angular de 22,5º com 10 lags e distâncias de $500 \mathrm{~m}$. Ajustou-se a esse variograma um modelo teórico igualmente exponencial, entretanto com efeito pepita de 100, patamar de 750 e alcance de $2.600 \mathrm{~m}$, representado por círculos pretos e linha tracejada preta.

A significativa diferença na altura dos patamares das duas direções (475 e 750) indica a presença de anisotropia zonal. Para modelar essa anisotropia, fez-se o cálculo de um modelo variográfico médio, considerando as duas direções, e obtendo para o efeito pepita o menor valor entre as duas direções, 612,5 para o patamar e $2.575 \mathrm{~m}$ para o alcance, para um modelo igualmente exponencial (triângulos pretos e linha preta contínua). A Figura 5 apresenta a modelagem variográfica descrita.

Os variogramas experimentais dos valores de concentração de $\mathrm{Pb}$ no solo, transformados para valores indicadores, também evidenciaram uma anisotropia zonal com estrutura semelhante à dos valores originais. Foi ajustado, para ambas as direções, um modelo de curva exponencial apresentando menor variabilidade na direção NE-SW $60^{\circ}$, com parâmetros de ajuste para um efeito pepita de 0,00055 , patamar de 0,0098 e alcance de $2.200 \mathrm{~m}$ (losango com linha preta tracejada). Para a direção NWSE $145^{\circ}$, de maior variabilidade, foi ajustado um modelo de curva igualmente exponencial, com efeito pepita de 0,001, patamar 0,0126 e alcance de $3.000 \mathrm{~m}$ (círculos pretos com linha preta tracejada). A modelagem para a anisotropia zonal presente revelou um variograma médio com efeito pepita igual a 0,000775, patamar de 0,0112 e alcance de $2.600 \mathrm{~m}$ (linha preta contínua). A Figura 6 apresenta esses variogramas descritos.

Definidos os parâmetros variográficos, foi efetuado o cálculo das estimativas pelo método
Figura 5

Variogramas direcionais e médio para $\mathrm{Pb}$.

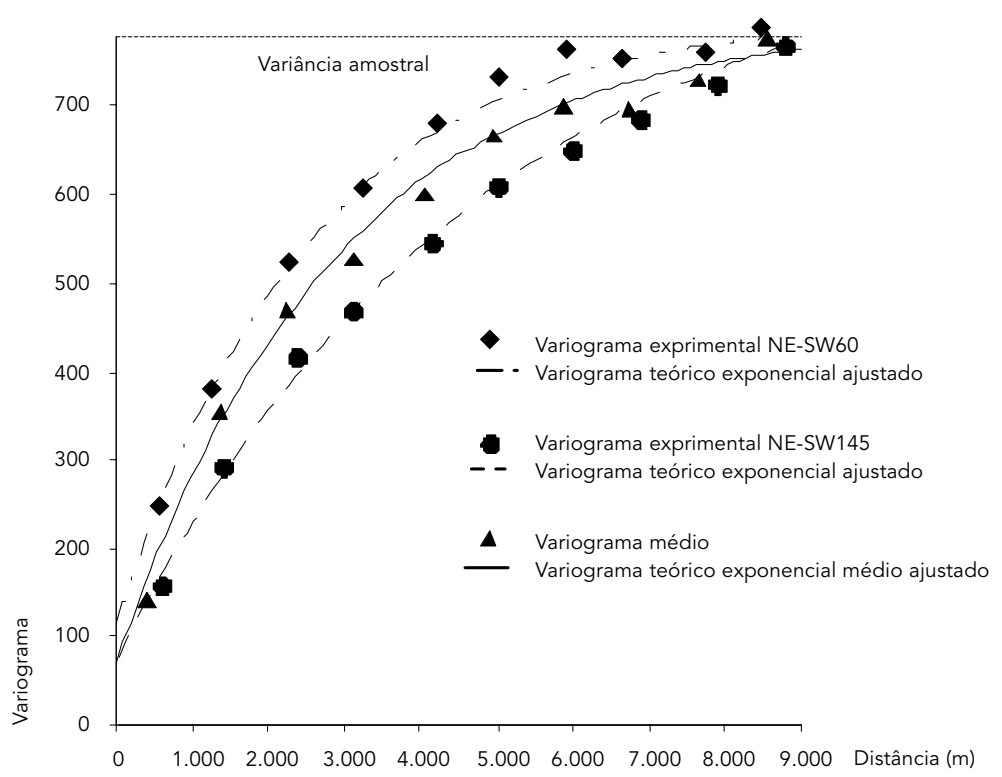

Figura 6

Variogramas indicativos.

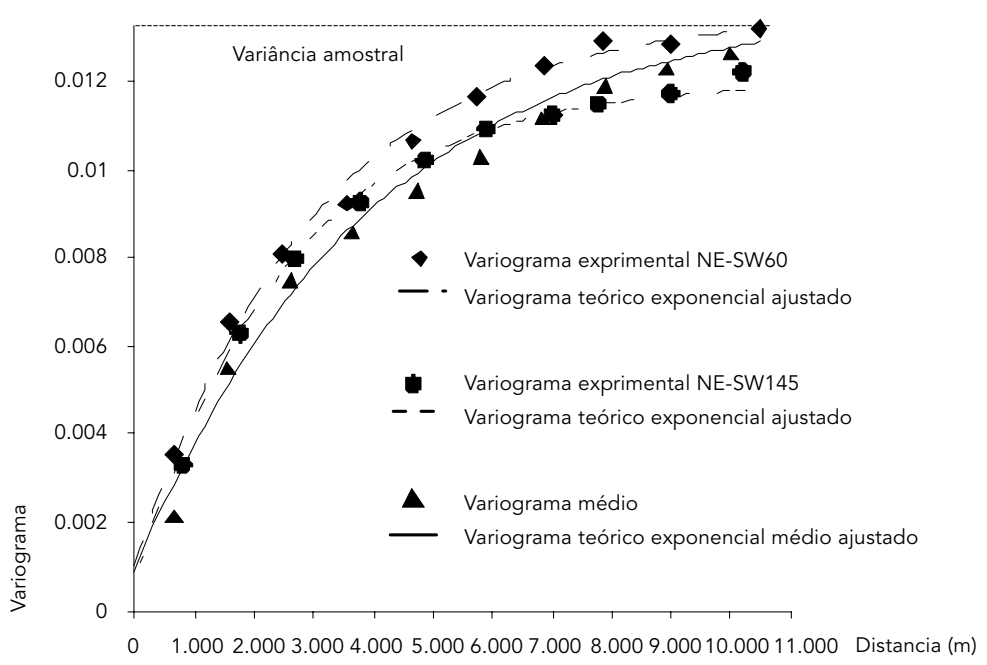


da KO, que resultou no mapa da distribuição espacial das concentrações do $\mathrm{Pb}$ (Figura 7).

O mapa da distribuição do $\mathrm{Pb}$ da Figura 7 apresenta faixas de áreas de poluição que se encontram em regiões com forte presença populacional. As mais significativas estão na parte centro-sul e leste do mapa. Entretanto, o setor com as maiores concentrações estão a noroeste do mapa, onde a região se caracteriza pela forte concentração industrial e intensa rede viária.

O mapa mostrando as áreas de maior risco à saúde pública, com indicação dos locais com maiores probabilidades de contaminação do solo por $\mathrm{Pb}$, obtidas por meio da KI, é apresentado na Figura 8. Nesse mapa, observam-se as áreas com variação de 0 a $100 \%$ de probabilidade de risco à saúde pública, o que permite evidenciar com maior propriedade as áreas com maiores chances de contaminação, oferecendo risco eminente à sua exposição. Os setores sul e sudeste mostram faixas de áreas com fortes concentrações de poluição, acompanhando em grande parte as instalações industriais e por muitas vezes a faixa de praia, como a parte centro-sul do mapa. No setor sudoeste, são evidenciadas mais três faixas antes não detectadas, associadas a pequenos cursos d'água e também à rede viária. Todavia, é novamente o setor noroeste que se apresenta com maiores concentrações, fortemente associado aos setores industriais, bem como às estradas e à rede de drenagem. Essa configuração, mostrada de modo não muito evidente no mapa da Figura 7 , acha-se ressaltada com relação ao estado de deterioração ambiental no mapa da Figura 8.

\section{Conclusões}

O conhecimento prévio de áreas com altos índices de poluição pode ser uma ferramenta útil nos estudos que considerem a relação entre os

Figura 7

Mapa das concentrações de $\mathrm{Pb}$

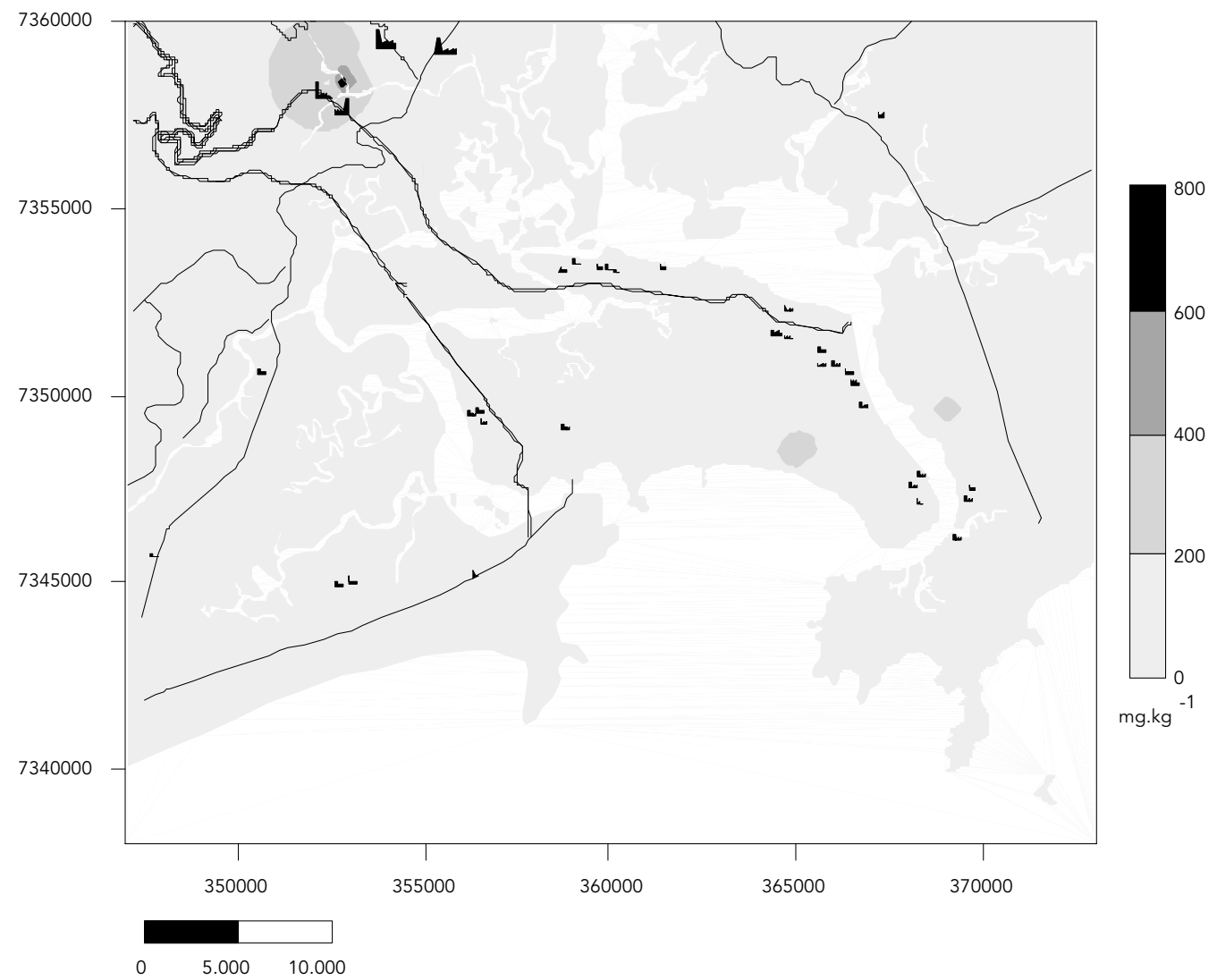




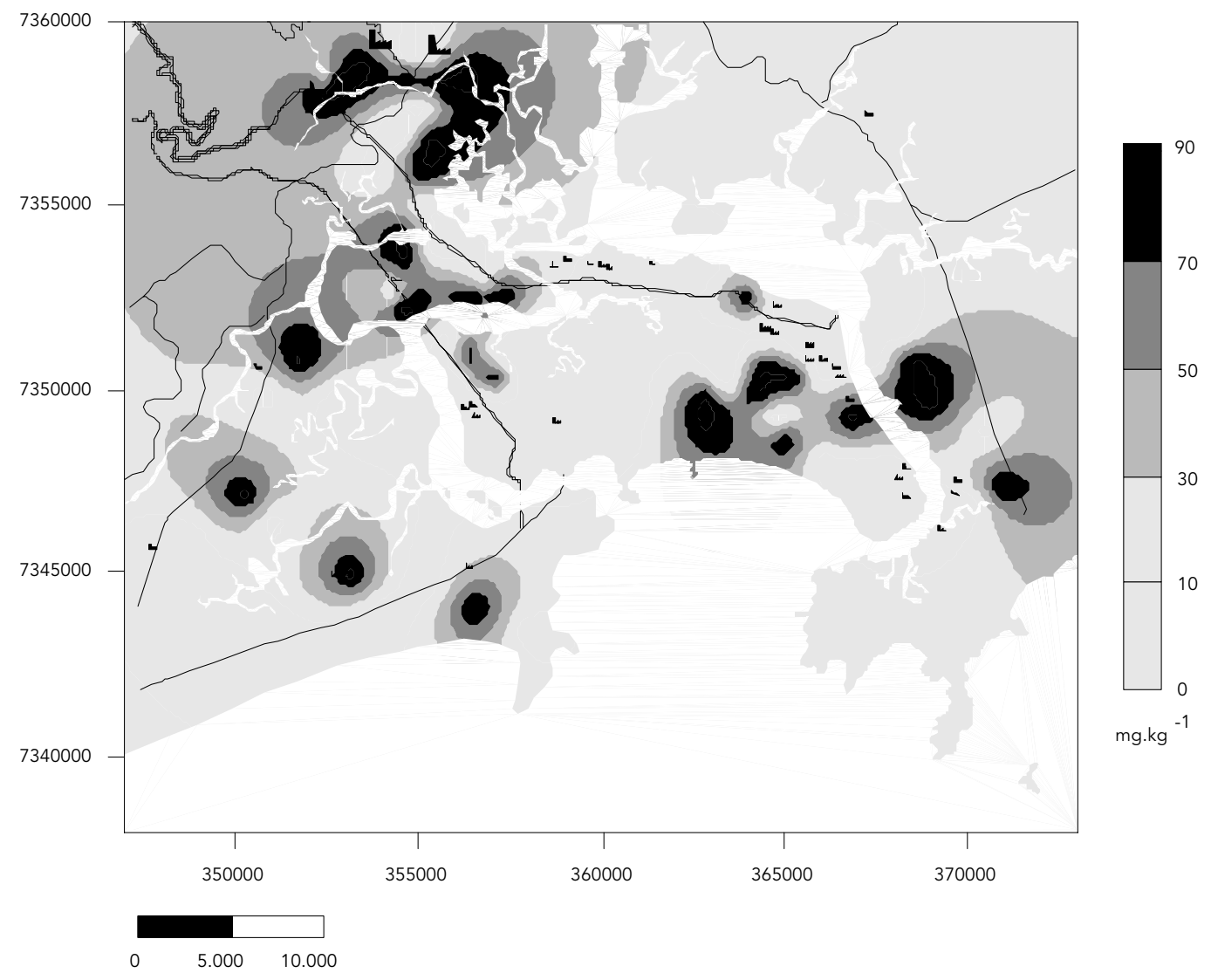

agentes nocivos à saúde humana e o meio ambiente.

A aplicação de técnicas de estatística espacial, como mostrado neste trabalho, justificase pelo fato de ser um importante instrumento de análise nos estudos de avaliação dos impactos observados sobre determinadas áreas. Com relação aos métodos geoestatísticos, estes têm sido cada vez mais utilizados por pesquisadores de diversas áreas do conhecimento, oferecendo uma importante contribuição nos estudos sobre mapeamento e saúde pública.

\section{Resumo}

Este trabalho tem por objetivo apresentar uma aplicação de métodos geoestatísticos na elaboração de mapas de risco à saúde pública, por meio da identificação de áreas com maior concentração de metais pesados. Foi escolhido o elemento chumbo $(\mathrm{Pb})$, resultante do transporte aéreo ou do carregamento das partículas causado pela lixiviação do solo, em uma região com grande concentração urbana e industrial na Baixada Santista, São Paulo, Brasil. Elaboraram-se mapas das distribuições espaciais desse elemento por intermédio da krigagem ordinária; posteriormente, utilizando-se a krigagem indicativa, identificaram-se as áreas com valores de contaminação do solo superiores aos níveis máximos aceitáveis pelo órgão de controle ambiental do Estado de São Paulo, originando um mapeamento com áreas com maior probabilidade de risco à saúde pública. Os mapas resultantes mostraram-se ferramentas promissoras para auxiliar a tomada de decisão quanto a questões de políticas públicas relacionadas à saúde e ao planejamento ambiental.

Metais Pesados; Mapa de Risco; Poluentes do Solo 


\section{Colaboradores}

R. W. Lourenço foi responsável pela redação, procedimentos de coleta e amostragem de campo, revisão da literatura, tratamento cartográfico e geoestatístico com análise dos resultados e conclusão. P. M. B. Landim foi responsável pela revisão, procedimentos de aplicação metodológica, tratamento geoestatístico com análise dos resultados e conclusão.

\section{Referências}

1. Moreira-Nodermann LM. A geoquímica e o meio ambiente. Geoquímica Brasilienses 1987; 1:89107.

2. Companhia de Tecnologia de Saneamento Ambiental. Manual de gerenciamento de áreas contaminadas. São Paulo: Companhia de Tecnologia de Saneamento Ambiental; 1999.

3. Atteia O, Dubois JP, Webster R. Geostatistical analysis of soil contamination in the Swiss Jura. Environ Poll 1994; 86:315-27.

4. Franssen A, van Eijnsbergen C, Stein A. Use of spatial prediction techniques and fuzzy classification for mapping soil pollutants. Geoderma 1997; 77: 243-62.

5. Carlon C, Critto A, Marcomini A, Nathanail P. Risk based characterisation of contaminated industrial site using multivariate and geostatistical tools. Environ Poll 2001; 111:417C-27.

6. van Meirvenne M, Goovaerts P. Evaluating the probability of exceeding a site-specific soil cadmium contamination threshold. Geoderma 2001; 102:75-100M.

7. Lin Y, Chang T, Teng T. Characterization of soil lead by comparing sequential Gaussian simulation, simulated annealing simulation and kriging methods. Environmental Geology 2001; 41:189-99.

8. Lin Y, Chang T, Shih C, Tseng C. Factorial and indicator kriging methods using a geographic information system to delineate spatial variation and pollution sources of soil heavy metals. Environmental Geology 2002; 42:900-9.

9. Critto A, Carlon C, Marcomini A. Characterization of contaminated soil and groundwater surrounding an illegal landfill (S. Giuliano, Venice, Italy) by principal component analysis and kriging. Environ Poll 2003; 122:235-44.

10. Juang K, Chen Y, Lee D. Using sequential indicator simulation to assess the uncertainty of delineating heavy-metal contaminated soils. Environ Poll 2004; 127:229-38.

11. McGrath D, Zhang C, Carton OT. Geostatistical analyses and hazard assessment on soil lead in Silvermines area, Ireland. Environ Poll 2004; 127: 239-48.

\section{Agradecimentos}

Agradecimentos à Fundunesp pelo auxílio na publicação e à Fundação de Amparo à Pesquisa do Estado de São Paulo (FAPESP) pelo financiamento do projeto de doutorado (processo 98/01162-2), do qual este trabalho fez parte. Agradecimentos, também, aos dois revisores pelas sugestões e críticas apresentadas.
12. American Society for Testing and Materials. Standard guide for risk-based corrective action applied at petroleum release sites. Annual book. West Conshohocken: American Society for Testing and Materials; 1995.

13. Swartjes FA. Risk-based assessment of soil and groundwater quality in the Netherlands: standards and remediation urgency. Risk Anal 1999; 19:1235-49.

14. Theelen RMC, Nijhof AG. Dutch methodology of risk assessment of contaminated soils. In: Contaminated Soils, 3rd International Conference on the Geochemistry of Trace Elements. Paris: Institut National de la Recherche Agronomique; 1997. p. 425-32.

15. Companhia de Tecnologia de Saneamento Ambiental. Estabelecimento de padrões de referência de qualidade e valores de intervenção para solos e águas subterrâneas no Estado de São Paulo. Relatório Parcial. São Paulo: Companhia de Tecnologia de Saneamento Ambiental; 1997.

16. Oliver MA, Webster R. Kriging: a method of interpolation for geographical information systems. International Journal of Geographic Information Systems 1990; 4:315-32.

17. Journel AG. Non-parametric estimation of spatial distribution. Mathematical Geology 1983; 15:44568.

18. Isaaks E, Srivastava R. An introduction to applied geostatistics. Oxford: Oxford University Press; 1989.

19. Sturaro JR, Landim PMB. Geoestatística indicativa aplicada à análise espacial. In: VII Simpósio Latinoamericano de Percepción Remota. Resumos Expandidos. México DF: La Sociedad Latinoamericana de Percepción Remota y Sistemas de Información Espacial; 1996. p. 68-73.

20. Sturaro JR, Landim PMB, Riedel PS. O emprego da técnica geoestatística da krigagem indicativa em geotecnia ambiental. Revista Solos e Rochas 2000; 23:157-64.

Recebido em 14/Jan/2004

Versão final reapresentada em 17/Mai/2004 Aprovado em 20/Jul/2004 